\title{
Multiple Images of a Highly Magnified Supernova Formed by an Early-Type Cluster Galaxy Lens
}

\author{
Patrick L. Kelly ${ }^{1 *}$, Steven A. Rodney ${ }^{2}$, Tommaso Treu ${ }^{3}$, Ryan J. Foley ${ }^{4}$, \\ Gabriel Brammer ${ }^{5}$, Kasper B. Schmidt ${ }^{6}$, Adi Zitrin ${ }^{7}$, Alessandro Sonnenfeld ${ }^{3}$, \\ Louis-Gregory Strolger ${ }^{5,8}$, Or Graur ${ }^{9}$, Alexei V. Filippenko ${ }^{1}$, Saurabh W. Jha ${ }^{10}$, \\ Adam G. Riess ${ }^{2,5}$, Marusa Bradac ${ }^{11}$, Benjamin J. Weiner ${ }^{12}$, Daniel Scolnic ${ }^{2}$, \\ Matthew A. Malkan ${ }^{3}$, Anja von der Linden ${ }^{13}$, Michele Trenti ${ }^{14}$, Jens Hjorth ${ }^{13}$, \\ Raphael Gavazzi ${ }^{15}$, Adriano Fontana ${ }^{16}$, Julian Merten ${ }^{7}$, Curtis McCully ${ }^{6}$, \\ Tucker Jones ${ }^{6}$, Marc Postman ${ }^{5}$, Alan Dressler ${ }^{17}$, Brandon Patel ${ }^{10}$, S. Bradley Cenko ${ }^{18}$, \\ Melissa L. Graham ${ }^{1}$, and Bradley E. Tucker ${ }^{1}$ \\ ${ }^{1}$ Department of Astronomy, University of California, Berkeley, CA 94720 \\ ${ }^{2}$ Department of Astronomy, The Johns Hopkins University, Baltimore, MD 21218 \\ ${ }^{3}$ Department of Physics and Astronomy, University of California, Los Angeles, CA 90095 \\ ${ }^{4}$ University of Illinois at Urbana-Champaign, 1002 W. Green Street, Urbana, IL 61801 \\ ${ }^{5}$ Space Telescope Science Institute, 3700 San Martin Drive, Baltimore, MD 21218 \\ ${ }^{6}$ Department of Physics, University of California, Santa Barbara, CA 93106 \\ ${ }^{7}$ California Institute of Technology, MC 249-17, Pasadena, CA 91125 \\ ${ }^{8}$ Western Kentucky University, 1906 College Heights Blvd., Bowling Green, KY 42101 \\ ${ }^{9}$ CCPP, New York University, 4 Washington Place, New York, NY 10003 \\ ${ }^{10}$ Rutgers, The State University of New Jersey, Piscataway, NJ 08854 \\ ${ }^{11}$ Department of Physics, University of California, Davis, CA 95616 \\ ${ }^{12}$ Steward Observatory, University of Arizona, Tucson, AZ 85721 \\ ${ }^{13}$ Dark Cosmology Centre, Juliane Maries Vej 30, 2100 Copenhagen, Demark \\ ${ }^{14}$ School of Physics, University of Melbourne, VIC 3010, Australia \\ ${ }^{15}$ Institut d'Astrophysique de Paris, 98 bis Boulevard Arago, F-75014 Paris, France \\ ${ }^{16}$ INAF-OAR, Via Frascati 33, 00040 Monte Porzio - Rome, Italy \\ ${ }^{17}$ Carnegie Observatories, 813 Santa Barbara Street, Pasadena, CA 91101 \\ ${ }^{18}$ NASA/Goddard Space Flight Center, Code 662, Greenbelt, MD 20771 \\ ${ }^{*}$ To whom correspondence should be addressed; E-mail: pkelly@astro.berkeley.edu.
}


We report the discovery of the first multiply-imaged gravitationallylensed supernova. The four images form an Einstein cross with over $2^{\prime \prime}$ diameter around a $z=0.544$ elliptical galaxy that is a member of the cluster MACS J1149.6+2223. The supernova appeared in Hubble Space Telescope near-infrared exposures taken on 3-20 November 2014 UT, as part of the Grism Lens-Amplified Survey from Space. The images of the supernova coincide with the strongly lensed arm of a spiral galaxy at $z=1.491$, which is itself multiply imaged by the cluster potential. A measurement of the time delays between the multiple images and their magnification will provide new unprecedented constraints on the distribution of luminous and dark matter in the lensing galaxy and in the cluster, as well as on the cosmic expansion rate.

The possibility that the light from an exploding supernova (SN) could follow more than a single path around an intervening strong galaxy lens to the observer was first explored exactly fifty years ago (1). Many decades of transient searches, however, have not identified an example of a SN visible at multiple positions around a gravitational lens. Here we report the discovery of the first strongly lensed SN found in resolved multiple images, which we identified in the MACS J1149.6+2223 (2) galaxy cluster field on 11 November $2014^{1}$.

The detection of a multiple-image transient provides the opportunity to measure two quantities of great cosmological interest. First, the difference in arrival time, owing to the difference in geometric and gravitational time delay (3), is directly proportional to the so-called time-delay distance and thus inversely proportional to the Hubble constant and weakly dependent on other cosmological parameters $(1,4-6)$. Second, for an assumed cosmological model, the time delays are a direct measurement of the difference in gravitational potential between the multiple images and thus greatly improve the reconstruction of the mass distribution in the deflector. Furthermore, if the lensed SN were of Type Ia, light-curve calibration $(7,8)$ would make it possible to infer the intrinsic brightness of the explosion with $\sim 10 \%$ precision. Comparison of the SN intrinsic brightness and its apparent brightness would then enable an estimate of the absolute magnifications experienced by the SN across the multiple images $(9,10)$. This in turn provides separate, powerful constraints on the lensing mass distribution (11, 12), and the cosmic expansion and geometry (13), breaking the mass-sheet degeneracy (14).

This supernova (hereafter 'SN Refsdal') is the first multiply imaged SN discovered to date. It was recently shown that PS1-10afx (15) is a redshift $z=1.38 \mathrm{SN}$ Ia strongly magnified (a factor of $\sim 30$ ) by an intervening galaxy at $z=1.12(16,17)$. Follow-up spectroscopy after the light from the SN had faded showed emission and absorption features produced by the $z=1.12$ galaxy lens. Since PS1-10afx was discovered and monitored

\footnotetext{
${ }^{1} \mathrm{UT}$ dates are used throughout this paper
} 
with ground-based telescopes, the available imaging had insufficient resolution to separate multiple potential images of the SN, and thus none of the applications described above could be pursued. For SN Refsdal, the four images are instead clearly resolved with an image separation of over $2^{\prime \prime}$, thus presenting an ideal opportunity to carry out for the first time the experiment suggested 50 years ago by Refsdal (1).

The Grism Lens-Amplified Survey from Space (GLASS) program (GO-13459; PI Treu) is a 140-orbit Hubble Space Telescope(HST) program that is obtaining near-infrared grism spectra of massive galaxy clusters with the primary goals of studying faint highredshift $(z \gtrsim 6)$ galaxies (18), spatially resolved intermediate-redshift galaxies $(19)$, and characterizing the cluster galaxy population. Wide-band near-infrared $F 105 \mathrm{~W}$ and $F 140 \mathrm{~W}$ exposures are taken using the Wide Field Camera 3 (WFC3) to align and calibrate the grism data, and we have been promptly searching these images for transient sources.

In the F140W GLASS images acquired on 10 November 2014, we detected the four component images of a quadruple lens system, which we label S1-S4. Figure 1 shows coadditions of 3-20 November $2014 \mathrm{~F} 105 \mathrm{~W}, \mathrm{~F} 125 \mathrm{~W}$, and $F 140 \mathrm{~W}$ images, as well as the difference between these images and pre-explosion exposures of the field acquired in 2010 and 2011. The fluxes we measure at the positions of S1-S4 from $F 105 W(3,8$, and 11 November 2014), F125W (20 November 2014), and F140W (10 and 20 November 2014) exposures are shown in Figure 2. While sources S1 and S2 do not show substantial changes in their fluxes, S3 exhibits a clear rise in brightness. The light curve of S4 is difficult to characterize with the currently available data, since it is comparatively faint.

Table 1 shows the coordinates of the variable sources. In Figure 3, the color-composite image shows the red galaxy lens at $z=0.544$ (20) surrounded by an Einstein ring formed by light from the distorted spiral host galaxy with $z=1.491$ (21), whose nucleus is offset by $\sim 3.3^{\prime \prime}$ from the center of the lensing elliptical galaxy.

The transient images are not coincident with the nucleus of the spiral host galaxy, making nuclear activity in the host an unlikely explanation. Indeed, the early-type galaxy lens only multiply images the tip of one of the spiral arms. A search of archival WFC3 $F 105 W, F 110 W, F 140 W$, and $F 160 W$ images of MACS J1149.6+2223 acquired across ten separate $H S T$ visits beginning on 4 December 2010 finds no evidence for previous variability. Figure S1 shows several epochs of registered and coadded F140W imaging having no significant variation - seven archival epochs of $F 160 \mathrm{~W}$ imaging likewise show no significant changes. Imaging without evidence for variability supports the hypothesis that the lensed source is an explosive transient, and not a flare from an active galactic nucleus (AGN) which would be expected to show long-term variability. We note that the transients detected on November 11 are several magnitudes above the upper limits obtained at previous epochs of $\sim 28.5 \mathrm{mag} \mathrm{AB}$. The amplitude of this variation would be very unusual for an $A G N$, whose light curves typically vary at the level of a few tenths of a magnitude over these time scales (20-24).

A separate possibility would be that the lensed source is an AGN not associated with the distorted spiral galaxy. However, if the hypothetical AGN had a different redshift 
from the spiral galaxy, the Einstein cross formed by the lensed sources would not be coincident with the Einstein ring formed by the blue light from the spiral (see Figure S2). Furthermore, such an AGN would likely have sufficient brightness for detection in the many previous exposures. Figure S1 reveals no point-like sources in earlier exposures spatially coincident with the multiple images we detect.

Given the configuration and the image separation, a significant fraction of the strong gravitational lensing potential is contributed by the massive galaxy. Because of its location near the critical lines of the cluster itself (25), there is also an important contribution from the overall cluster potential. This makes modeling of the system particularly complex, and owing to the time-critical nature of this report, full modeling is deferred for future studies.

In order to obtain a rough estimate of the time delay and magnification ratios between the images, we constructed fourteen preliminary mass models, by modeling the system with variations of a single power-law ellipsoid mass distribution plus an external shear. As expected for this symmetric configuration (26), the time delays are relatively short and the magnification ratios are of order one-to-a-few. As one example, the simple model consisting of a single isothermal ellipsoid embedded in a strong external shear (26) yields time delays of the order of several tens of days. S1 is generally the leading image, typically followed by S2, S3, and then S4. Magnifications are of the order 2 for the least magnified source $\mathrm{S} 4$ and of the order $\sim 10$ for the other images. These magnifications do not include the additional contribution from the cluster, which is expected to be significant. Earlier modeling has found a relatively flat, nearly convergent central mass distribution, evident from the nearly undistorted shape of the spiral images (25). Adding a sheet of high-density matter and hence convergence onto our simplistic galaxy-lens model is expected to boost magnifications. Our cluster lens model shows typical magnifications of $\sim 20-30$ in the area in which the lens galaxy is embedded (27). The short predicted time delays and small magnification ratios are consistent with our identification of the system as a multiply imaged SN, because we expect the luminosity of the SN not to vary dramatically over the time scale of less than a week in the rest frame.

The spiral host galaxy itself is multiply imaged by the galaxy cluster $(21,25)$, so the SN may be detected at future epochs, or may have already appeared elsewhere in the cluster field. A search of archival HST imaging in both the optical ( F606W, F814W, and $F 850 L P)$ and infrared $(F 105 \mathrm{~W}, F 125 \mathrm{~W}, F 140 \mathrm{~W}$, and $F 160 \mathrm{~W})$ at the locations of the multiple images of the presumed host galaxy has revealed no evidence for SN Refsdal when these data were taken. Our cluster lens model predicts that the SN will appear in the central image of the spiral host galaxy, at an approximate position of $\alpha=11^{\mathrm{h}} 49^{\mathrm{m}} 36.01^{\mathrm{s}}$, $\delta=+22^{\circ} 23^{\prime} 48.13$ (J2000.0), at a future time (about a decade from now).

Due to a favorable coincidence, Frontier Fields program observations (GO-11504; PI Lotz) of the MACS J1149.6+2223 galaxy cluster field began on 20 November 2014 and run through January 2015 with WFC3 F105W, F125W, F140W, and F160W broadband filters, supplemented with additional observations from the FrontierSN program (GO-13386,13790; PI Rodney), to sample the SN light curve with a reasonable cadence. These anticipated 
data will make it possible to measure the time delays among the multiple images from the light curve. If a spectrum having sufficient signal-to-noise ratio can be obtained using a ground- or space-based instrument, it will provide a useful constraint on the phase of the SN light in each image independent of measurements of its light curve. A HST Director's Discretionary time program (GO-14041; PI Kelly) will obtain multiple band optical data on the SN to constrain its spectroscopic type from its wide-band spectral energy distribution.

This is the first discovery of a $\mathrm{SN}$ whose light is bent by an intervening lens to form multiple, resolved images. Measurements of the time delays between the phase of the SN in the pairs of images will enable constraints on the foreground mass distribution, and on the expansion and geometry of the universe. If the SN is of Type Ia, separate, even stronger limits on both the matter distribution and cosmological parameters become possible.

Acknowledgements This work is based on data obtained with the NASA/ESA Hubble Space Telescope. We thank Ori Fox, WeiKang Zheng, Josh Bloom, Charles Keeton, Jon Mauerhan, Chuck Steidel, and Allison Strom for helpful discussions, as well as the Space Telescope Science Institute and Director Matt Mountain for supporting our proposal for follow-up observations. GLASS is supported by NASA through HST grant GO-13459. Support for SAR was provided by NASA through Hubble Fellowship grant HST-HF51312.01 awarded by the Space Telescope Science Institute, which is operated by the Association of Universities for Research in Astronomy, Inc., for NASA, under contract NAS 5-26555. Follow-up imaging through the FrontierSN program is supported by NASA through grant HST-GO-13386.001. A.V.F.'s group at UC Berkeley has received generous financial assistance from the Christopher R. Redlich Fund, the TABASGO Foundation, and NSF grant AST-1211916. The Dark Cosmology Centre is funded by the DNRF. Support for AZ was provided by NASA through Hubble Fellowship grant HST-HF2-51334.001-A awarded by STScI. Supernova research at Rutgers University is supported in part by NSF CAREER award AST-0847157 to SWJ. C.M is supported by the NSF Program 1313484

\section{References}

1. S. Refsdal, MNRAS 128, 307 (1964).

2. H. Ebeling, A. C. Edge, J. P. Henry, ApJ 553, 668-676 (2001).

3. I. I. Shapiro, Physical Review Letters 13, 789-791 (1964).

4. T. Treu, ARA\&A 48, 87-125 (2010).

5. E. V. Linder, Phys. Rev. D 84, 123529 (2011).

6. S. H. Suyu et al., ApJ 788, L35 (2014).

7. M. M. Phillips, ApJ 413, L105-L108 (1993). 


\begin{tabular}{ccc}
\hline Name & R.A. (J2000) & Dec (J2000) \\
\hline S1 & $11: 49: 35.574$ & $+22: 23: 44.26$ \\
S2 & $11: 49: 35.451$ & $+22: 23: 44.84$ \\
S3 & $11: 49: 35.369$ & $+22: 23: 43.95$ \\
S4 & $11: 49: 35.472$ & $+22: 23: 42.62$ \\
\hline
\end{tabular}

Table 1: Coordinates of the transient point sources detected around the cluster galaxy lens, in J2000 Right Ascension and Declination.

8. A. G. Riess, W. H. Press, R. P. Kirshner, ApJ 473, 88 (1996).

9. B. Patel et al., ApJ 786, 9 (2014).

10. J. Nordin et al., MNRAS 440, 2742-2754 (2014).

11. T. Riehm et al., A\&A 536, A94 (2011).

12. X. Li, J. Hjorth, J. Richard, JCAP 11, 15 (2012).

13. M. Oguri, Y. Kawano, MNRAS 338, L25-L29 (2003).

14. E. E. Falco, M. V. Gorenstein, I. I. Shapiro, ApJ 289, L1-L4 (1985).

15. R. Chornock et al., ApJ 767, 162 (2013).

16. R. M. Quimby et al., ApJ 768, L20 (2013).

17. R. M. Quimby et al., Science 344, 396-399 (2014).

18. K. B. Schmidt et al., ApJ 782, L36 (2014).

19. T. Jones et al., ArXiv e-prints, arXiv:1410.0967 (2014).

20. H. Ebeling et al., ApJ 661, L33-L36 (2007).

21. G. P. Smith et al., ApJ 707, L163-L168 (2009).

22. S. Kaspi et al., ApJ 659, 997-1007 (2007).

23. M. C. Bentz et al., ApJ 705, 199-217 (2009).

24. K. B. Schmidt et al., ApJ 714, 1194-1208 (2010).

25. A. Zitrin, T. Broadhurst, ApJ 703, L132-L136 (2009).

26. F. Courbin et al., A\&SA 536, A53 (2011).

2\%. A. Zitrin et al., ArXiv e-prints, arXiv:1411.1414 (2014). 

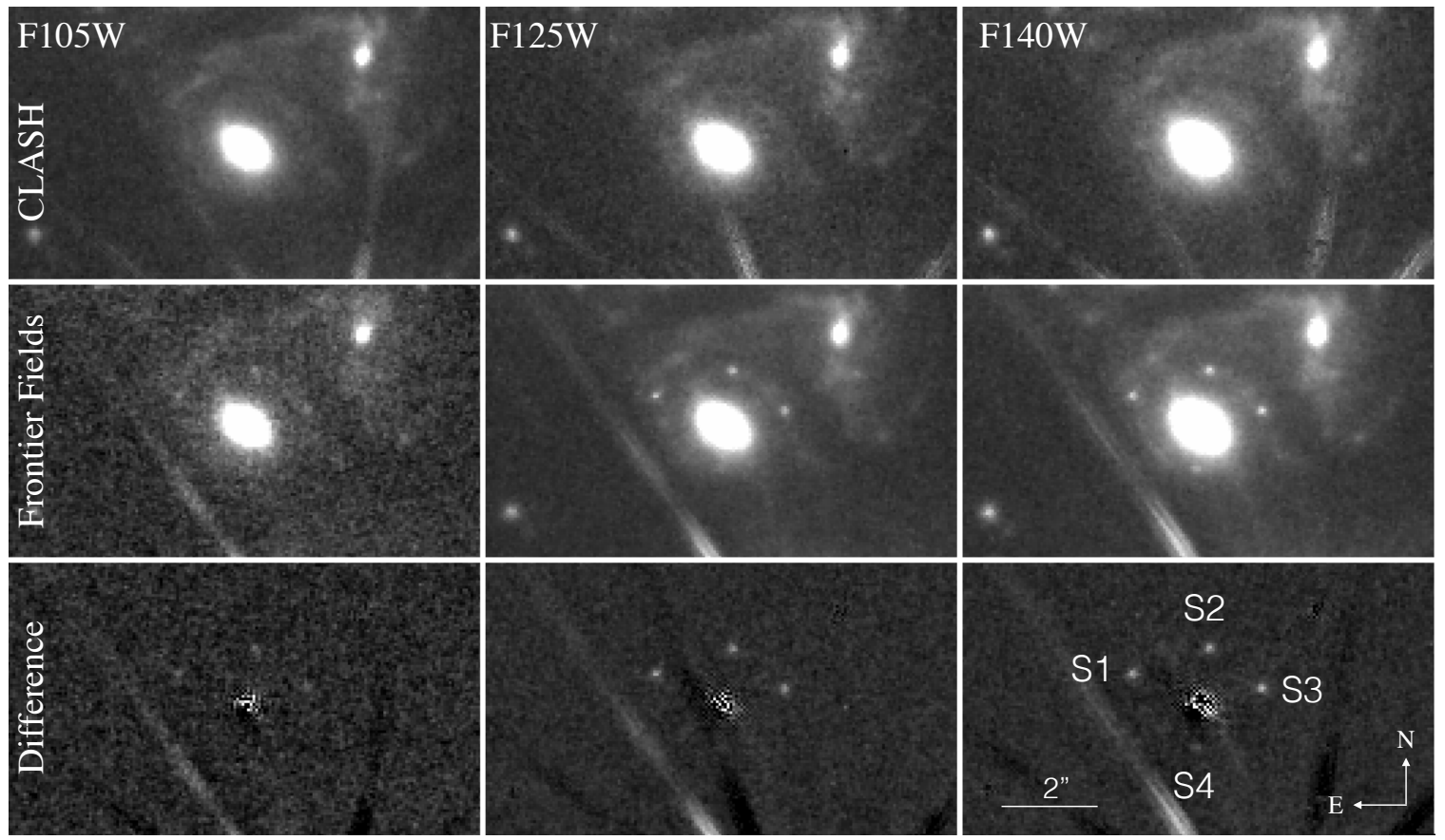

Fig. 1: $\quad H S T$ WFC3-IR images showing the simultaneous appearance of four point sources around a cluster member galaxy. From left to right the columns show imaging in the $F 105 \mathrm{~W}$ filter (Y band), F125W (J), and F140W (JH). From top to bottom the rows show archival imaging from the CLASH program, discovery epoch images from GLASS and the Hubble Frontier Fields programs, and the difference images. The template imaging in the top row comprises all available archival WFC3-IR imaging in these filters, collected from 05 December 2010 through 10 March 2011. The middle row of images are the composite of all available HST imaging collected between 3 November and 11 November 2014 (for $F 105 \mathrm{~W}$, at left), on 20 November 2014 (F125W, middle), and between 10 November and 20 November 2014 (F140W, right). The sources S1, S2, S3, and S4, which form an Einstein cross, are absent from all images obtained at earlier epochs, and therefore are clearly detected in the difference images along the bottom row. The line segments below S4 and in the lower-right corner are diffraction spikes from a nearby bright star in the foreground. 


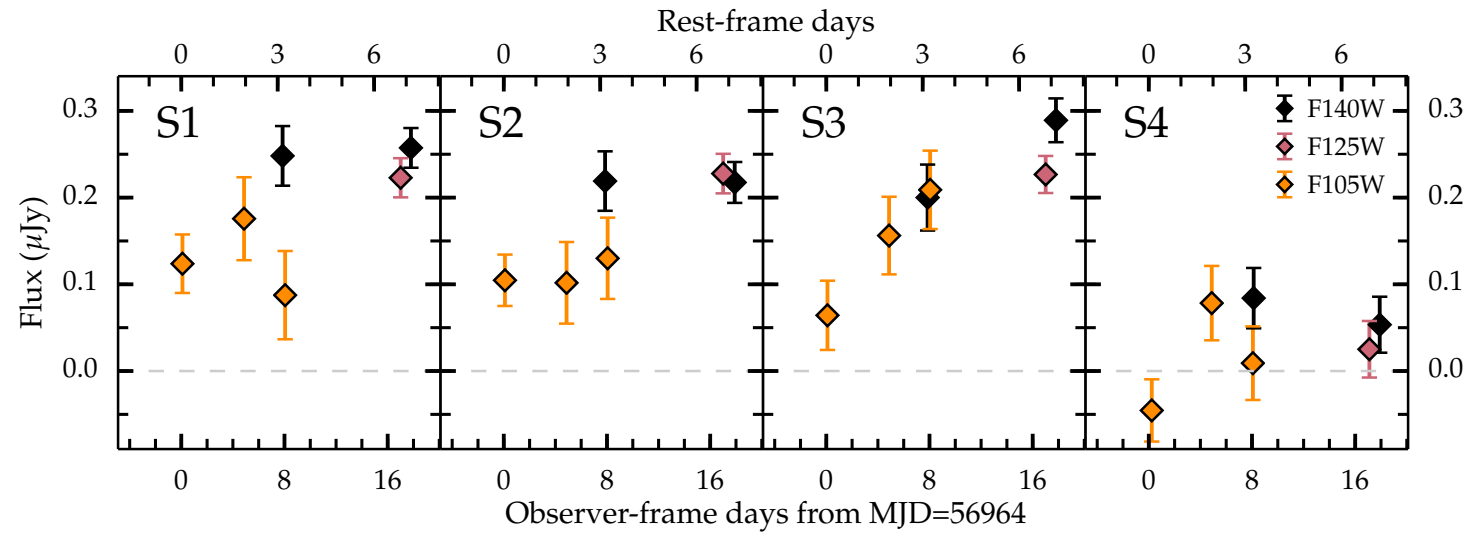

Fig. 2: Light curve of the images S1-S4 of the strongly lensed SN taken from 3 November 2014 through 20 November 2014. Rest-frame days assume the supernova is at the redshift of the multiply imaged spiral galaxy $(\mathrm{z}=1.491)$. We plot the fluxes measured in the WFC3 $F 105 \mathrm{~W}, F 125 \mathrm{~W}$, and F140W images of the MACS J1149.6+2223 galaxy cluster field. The expected time delays between images of days to weeks suggest that the transient must evolve over a timescale similar to that of a SN. A generic prediction of our lens models is that images $\mathrm{S} 2, \mathrm{~S} 3$, and $\mathrm{S} 4$ are predicted to be delayed relative to $\mathrm{S} 1$, which is consistent with the early photometry. 

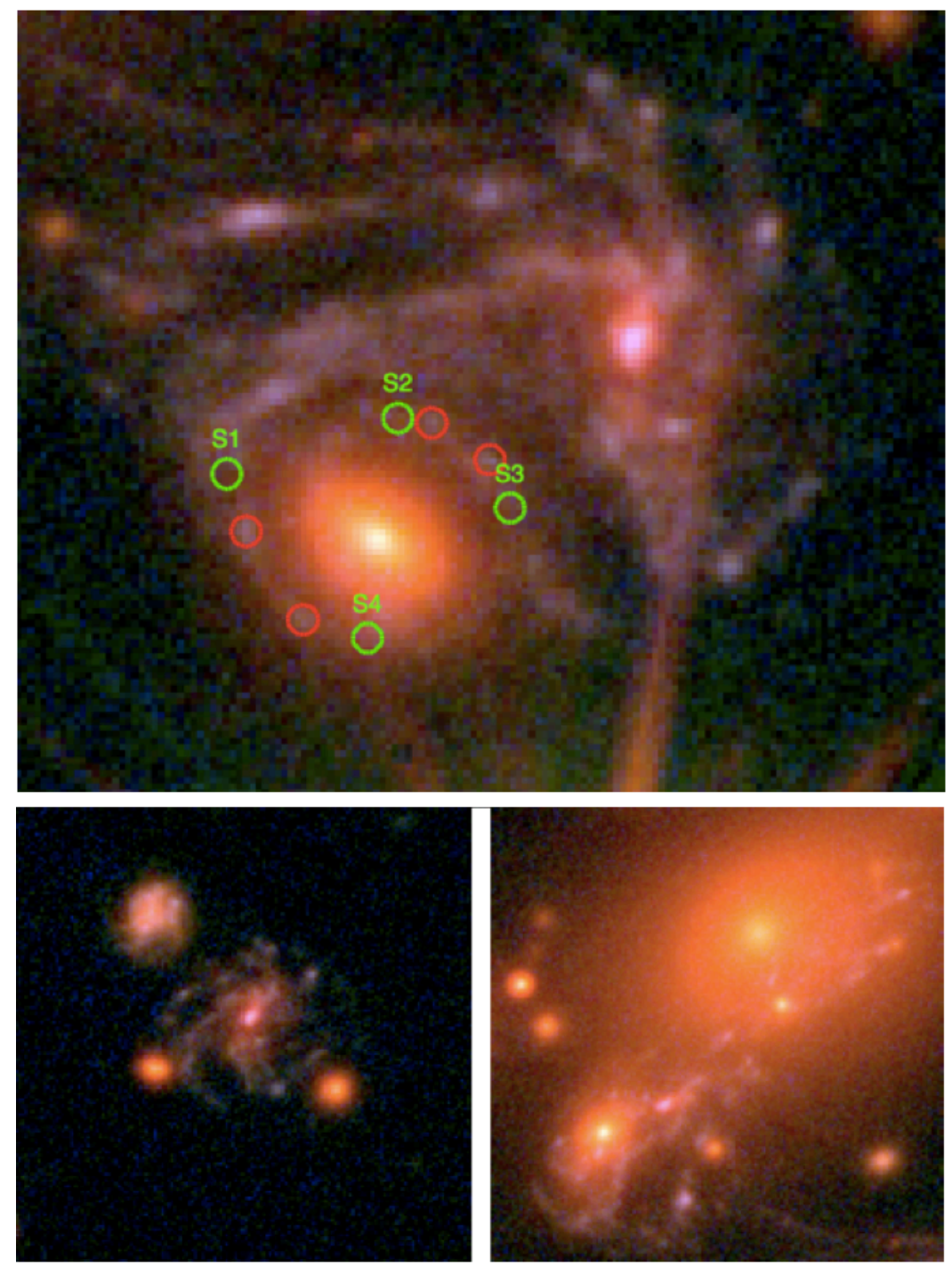

Fig. 3: Color-composite image of lensing elliptical galaxy and distorted background $(z=1.491)$ host spiral (top). The nucleus of the bluer host galaxy is offset by $\sim 3.3^{\prime \prime}$ from the red lensing elliptical galaxy $(z=0.544)$, suggesting the detected sources are separate from any possible nuclear activity in the host spiral galaxy. The green circles show the locations of images S1-S4, while another quadruply imaged segment of the spiral arm is marked in red (28). The orange line segments at the bottom of the upper panel are diffraction spikes from a nearby star. The spiral galaxy itself is known to be multiply imaged by the cluster $(21,25)$. The bottom panels show two additional lensed images of the spiral host galaxy visible in the galaxy cluster field. 

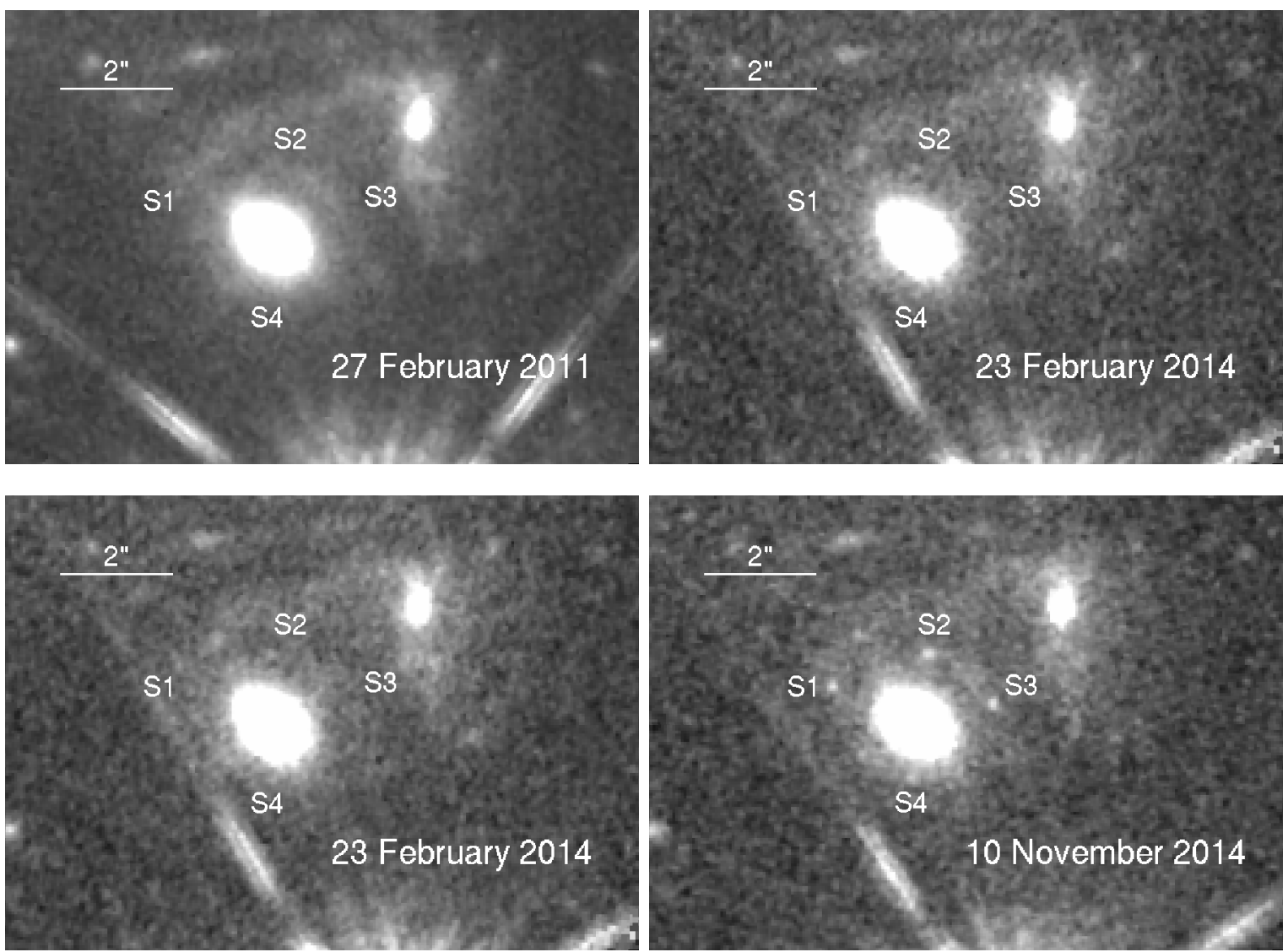

Fig. S1: Images of the lensing system from archival HST WFC3-IR observations in the F140W filter. All exposures obtained prior to 3 November 2014 show no evidence for variability at any of the positions associated with SN Refsdal.

\section{Supporting Online Material}

Previous and Future Appearances of SN Refsdal in Multiple Images of its Host Galaxy Our cluster lens model (27) predicts that SN Refsdal might have appeared some $\sim 20$ years ago in spiral image labeled 1.1 in Zitrin \& Broadhurst (2007; hereafter ZB07). The SN may additionally have appeared a couple of years ago in spiral images ZB07 1.3/1.4 (25, 28), or, alternatively, will appear there in approximately a decade. Our model also suggests that SN Refsdal could have appeared some $\sim 40-50$ years ago in spiral image ZB07 1.5 (25), although the SN position is believed not to be present in this multiple image (28). These estimates are highly approximate, because time delays on these cluster scales depend very sensitively on the exact expected position, and a fraction of the images are only partial. 


\begin{tabular}{ccccc}
\hline Date & MJD & Filter & Exposure Time (sec) & HST Program ID \\
\hline 2004 Apr 22.7 & 53117.7 & $F 814 W$ & 4590 & 9722 \\
2006 May 25.5 & 53880.5 & $F 814 W$ & 2184 & 10493 \\
2010 Dec 04.9 & 55534.9 & $F 850 L P$ & 1032 & 12068 \\
2010 Dec 05.0 & 55535.0 & $F 125 W$ & 1509 & 12068 \\
2010 Dec 05.0 & 55535.0 & $F 160 W$ & 1006 & 12068 \\
2011 Jan 16.0 & 55577.0 & $F 110 W$ & 1509 & 12068 \\
2011 Jan 16.0 & 55577.0 & $F 606 W$ & 1032 & 12068 \\
2011 Jan 16.0 & 55577.0 & $F 850 L P$ & 1044 & 12068 \\
2011 Jan 16.1 & 55577.1 & $F 160 W$ & 1006 & 12068 \\
2011 Jan 30.6 & 55591.6 & $F 850 L P$ & 1032 & 12068 \\
2011 Jan 30.7 & 55591.7 & $F 105 W$ & 1509 & 12068 \\
2011 Jan 30.7 & 55591.7 & $F 140 W$ & 1006 & 12068 \\
2011 Feb 13.3 & 55605.3 & $F 625 W$ & 1015 & 12068 \\
2011 Feb 27.0 & 55619.0 & $F 850 L P$ & 1032 & 12068 \\
2011 Feb 27.1 & 55619.1 & $F 775 W$ & 994 & 12068 \\
2011 Feb 27.1 & 55619.1 & $F 160 W$ & 1509 & 12068 \\
2011 Feb 27.1 & 55619.1 & $F 110 W$ & 906 & 12068 \\
2011 Feb 27.5 & 55619.5 & $F 606 W$ & 1032 & 12068 \\
2011 Feb 27.6 & 55619.6 & $F 140 W$ & 1306 & 12068 \\
2011 Feb 27.7 & 55619.7 & $F 105 W$ & 1306 & 12068 \\
2011 Mar 09.8 & 55629.8 & $F 160 W$ & 1509 & 12068 \\
2011 Mar 09.8 & 55629.8 & $F 625 W$ & 1032 & 12068 \\
2011 Mar 09.8 & 55629.8 & $F 775 W$ & 1053 & 12068 \\
2011 Mar 09.9 & 55629.9 & $F 125 W$ & 1006 & 12068 \\
2013 Nov 02.1 & 56598.1 & $F 160 W$ & 5508 & 13504 \\
2014 Feb 23.4 & 56711.4 & $F 105 W$ & 762 & 13459 \\
2014 Feb 23.7 & 56711.7 & $F 140 W$ & 762 & 13504 \\
2014 Feb 23.9 & 56711.9 & $F 105 W$ & 406 & \\
2014 Feb 25.6 & 56713.6 & $F 105 W$ & 762 & \\
2014 Apr 14.7 & 56761.7 & $F 814 W$ & 5246 & \\
\hline
\end{tabular}

Table S1: Past epochs of HST imaging on the MACS J1149.6+2223 field. In a visual inspection of difference images constructed from these data, no evidence for any transient source in any image of the SN Refsdal host galaxy was found. 
Absence of Variability in Prior Imaging. To check for past episodes of variability from this source, we performed a visual search of difference images constructed from archival HST imaging in both the optical (F435W, F606W, F814W, and F850LP) and IR (F105W, $F 125 \mathrm{~W}, F 140 \mathrm{~W}$, and $F 160 \mathrm{~W})$. We found no evidence of any transient sources prior to 3 November 2014 at any of the positions S1-S4 identified with SN Refsdal. There is also no variable source apparent at the locations of any of the multiple images of the presumed host galaxy elsewhere in the cluster field. A subset of the available IR images from previous $H S T$ observations are shown in Figure S1, and the full list of prior imaging epochs we evaluated in this search is given in Table S1.

Association of the SN with the Strongly Distorted Spiral Galaxy. Figure S2 shows that the broad-band spectral energy distribution (SED) of the Einstein ring that surrounds the four images of the SN matches that of the $z=1.491$ spiral galaxy, after subtracting contaminating light from the underlying elliptical galaxy lens. The subtraction is accomplished by scaling the SED of the early-type galaxy lens until its $F 140 \mathrm{~W}$ flux matches that measured for the Einstein Ring, and removing the scaled SED.

In Figure S3 we show a spectrum from the location of the Einstein Ring, collected with the Keck telescope in Hawaii on 20 November 2014. An emission feature at $9727 \AA$ is observed from knots in the Einstein Ring that are distinct from the supernova source positions, but spatially associated with the same ring feature. We identify this as [O II] $\lambda \lambda 3727$, giving further confirmation that the ring around the lensing galaxy is at $z=1.49$, the same redshift as the presumed spiral galaxy host behind the cluster. No emission from SN Refsdal is apparent in this spectrum.

Photometry of Multiple Images of the SN In Table S2, the magnitudes of the sources are shown for the three $F 105 \mathrm{~W}$, single $F 125 \mathrm{~W}$, and two $F 140 \mathrm{~W}$ visits, measured using point spread function fitting photometry.

\section{References}

28. S. Rau, S. Vegetti, S. D. M. White, MNRAS 443, 957-968 (2014). 

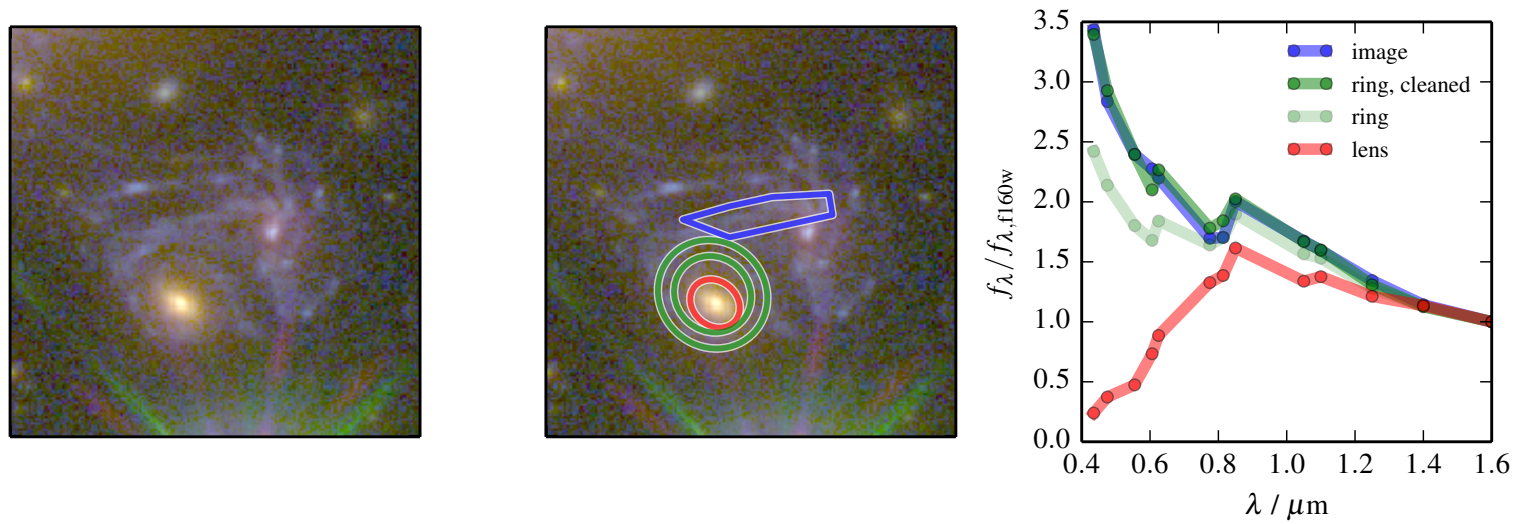

Fig. S2: Spectral energy distributions (SEDs) of the $z=1.491$ spiral host galaxy, Einstein ring, and elliptical galaxy lens measured from pre-explosion HST imaging. After subtracting the underlying light from the red, elliptical galaxy lens, the SED of the Einstein ring matches that of the strongly distorted $z=1.491$ spiral galaxy. This indicates that the SN, whose images are embedded in the Einstein ring, is spatially coincident with the spiral galaxy. Lens modeling also strongly suggests that the ring is associated with the main lensed galaxy (25). The blue polygon is an aperture enclosing the spiral host galaxy light, the green elliptical annulus aperture encloses the Einstein ring where the multiple SN images appeared, and the red elliptical aperture encloses the lens. All of the SEDs are normalized to the $F 160 \mathrm{~W}$ flux. 


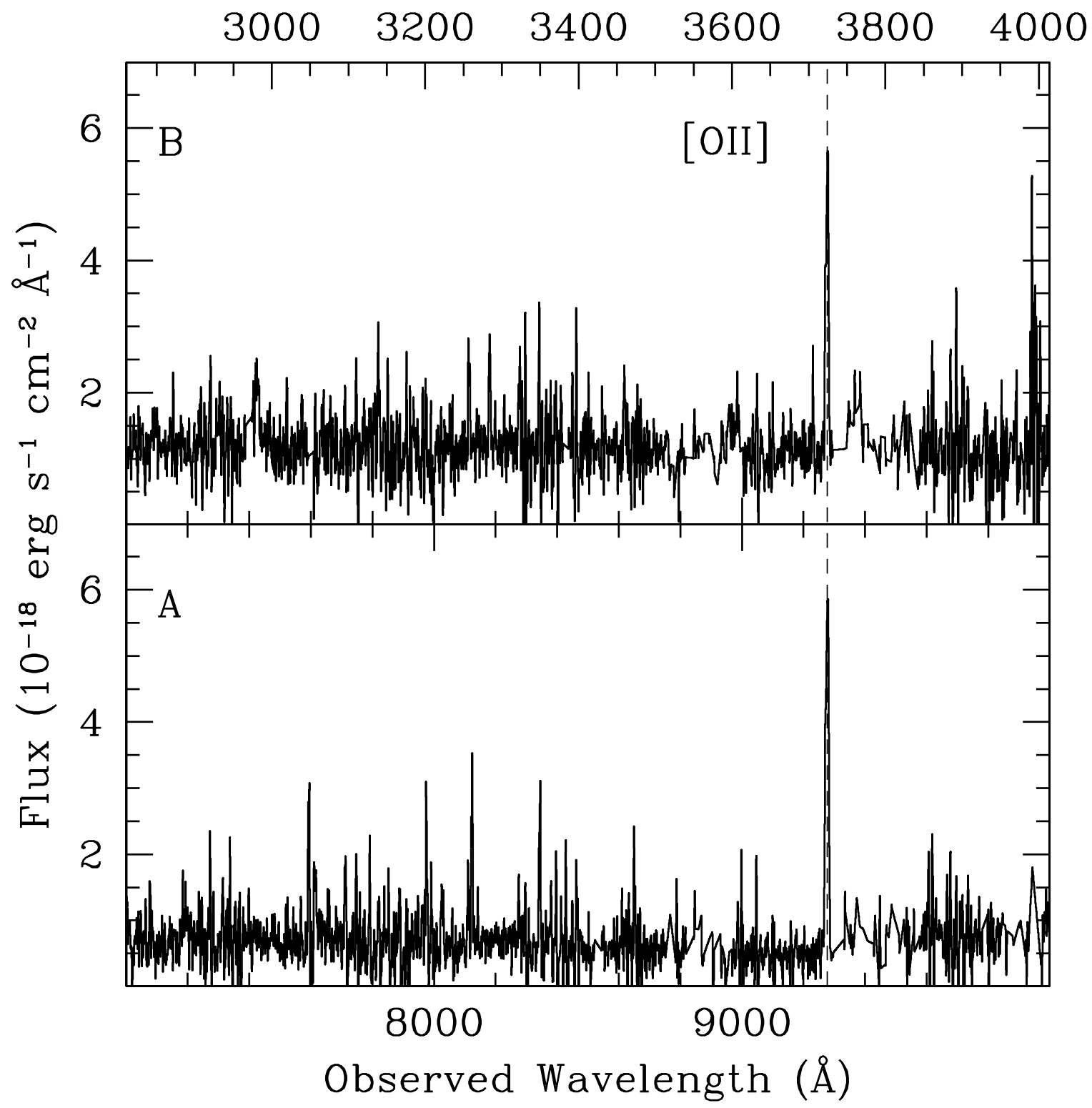

Fig. S3: A spectrum of the SN Refsdal host galaxy, taken with the Keck telescope's Low Resolution Imaging Spectrometer (LRIS) on 20 November 2014. For these observations the LRIS long slit was aligned along a portion of the Einstein ring, between the positions of sources S1 and S2. The [O II] $\lambda 3727$ emission line is clearly detected at an observer-frame wavelength of $9727 \AA$, confirming the redshift of the Einstein Ring at $z=1.49$. The top axis marks the rest-frame wavelength in Angstroms for this redshift solution. 


\begin{tabular}{crrrrrr}
\hline Source & MJD & Filter & Flux $(\mu$ Jy $)$ & Flux Err & AB Mag & Mag Err \\
\hline S1 & F140W & 56971.9 & 0.248 & 0.034 & 25.41 & 0.16 \\
S1 & F140W & 56981.8 & 0.257 & 0.023 & 25.38 & 0.14 \\
S1 & F125W & 56981.0 & 0.223 & 0.023 & 25.53 & 0.18 \\
S1 & F105W & 56964.1 & 0.124 & 0.034 & 26.16 & 0.30 \\
S1 & F105W & 56968.9 & 0.176 & 0.048 & 25.79 & 0.28 \\
S1 & F105W & 56972.1 & 0.087 & 0.051 & 26.46 & 0.43 \\
S2 & F140W & 56971.9 & 0.219 & 0.034 & 25.54 & 0.17 \\
S2 & F140W & 56981.9 & 0.217 & 0.024 & 25.55 & 0.12 \\
S2 & F125W & 56981.0 & 0.228 & 0.023 & 25.50 & 0.15 \\
S2 & F105W & 56964.1 & 0.105 & 0.030 & 26.33 & 0.30 \\
S2 & F105W & 56968.9 & 0.102 & 0.047 & 26.44 & 0.40 \\
S2 & F105W & 56972.1 & 0.130 & 0.047 & 26.03 & 0.36 \\
S3 & F140W & 56971.9 & 0.200 & 0.038 & 25.65 & 0.21 \\
S3 & F140W & 56981.8 & 0.289 & 0.025 & 25.26 & 0.12 \\
S3 & F125W & 56981.0 & 0.227 & 0.021 & 25.51 & 0.14 \\
S3 & F105W & 56964.1 & 0.064 & 0.040 & 26.93 & 0.62 \\
S3 & F105W & 56968.9 & 0.156 & 0.045 & 25.90 & 0.32 \\
S3 & F105W & 56972.1 & 0.209 & 0.045 & 25.67 & 0.27 \\
S4 & F140W & 56972.1 & 0.084 & 0.035 & 26.59 & 0.45 \\
S4 & F140W & 56981.9 & 0.053 & 0.032 & 27.08 & 0.66 \\
S4 & F125W & 56981.1 & 0.025 & 0.033 & 27.90 & 1.41 \\
S4 & F105W & 56964.2 & -0.045 & 0.036 & 26.70 & $\ldots$ \\
S4 & F105W & 56968.9 & 0.078 & 0.043 & 26.66 & 0.59 \\
S4 & F105W & 56972.1 & 0.009 & 0.042 & 29.01 & 5.10 \\
\hline
\end{tabular}

Table S2: HST WFC3-IR photometry. The observed fluxes were measured with point spread function fitting on difference images using template images constructed from all WFC3-IR images collected prior to November 2014. In cases where the source is not detected and noise from the sky results in a measurement of negative flux, we report the magnitude as a 3- $\sigma$ upper limit. 